\title{
Artroplastia total coxofemoral em cães. Estudo experimental com prótese nacional
}

\author{
Total hip arthroplasty in dogs. Experimental study using a prosthesis made in Brazil
}

\author{
Bruno Watanabe Minto ${ }^{I}$ Cláudia Valéria Seullner BrandãoII Gilberto José Cação Pereira'III \\ Paulo Vinicius Mortensen Steagall ${ }^{\text {II }}$ Maria Jaqueline Mamprim ${ }^{\text {III }}$ José Joaquim Titton Ranzani ${ }^{\text {II }}$
}

\section{RESUMO}

A prótese total da articulação coxofemoral representa uma das técnicas mais aceitas, nos Estados Unidos e na Europa, para o tratamento cirúrgico da displasia coxofemoral severa em cães. Entretanto, ainda é pouco difundida e estudada no Brasil. No presente trabalho, foi utilizada prótese confeccionada no Brasil, com o objetivo de avaliar a sua aplicação, evolução pós-operatória $e$ complicações associadas. Foram utilizados dez cães, sadios $e$ adultos. Todos foram submetidos ao procedimento de prótese total cimentada da articulação esquerda utilizando-se um componente femoral de cromo-cobalto, cabeça fixa, e uma cúpula acetabular de polietileno de alta densidade. As avaliações clínicas e radiográficas foram realizadas no préoperatório e aos 30, 60, 90, 120 e 150 dias após a intervenção cirúrgica. A prótese total utilizada proporcionou um bom resultado funcional ao membro operado, em $80 \%$ dos animais. As principais complicações relacionadas foram a luxação protética e a soltura do componente acetabular.

Palavras-chave: articulação coxofemoral, cão, prótese total.

\section{ABSTRACT}

Total hip prosthesis is one of the most accepted methods used in the United States and Europe for the treatment of severe hip dysplasia in dogs. However, there are few studies with the technique and the procedure is still not well established in Brazil. A prosthesis made in Brazil was used. The purpose of this study was to evaluate its application and determine the postoperative finds and complications. Ten mature healthy dogs were used. A fixed-head cemented total hip prosthesis was applied on the left leg of the dogs. A chrome-cobalt femoral stem and high density polyetylene acetabular cup was used.
Clinical and radiographic evaluations were performed before surgery and again at 30, 60, 90, 120 e 150 days post operation. Proper weight bearing was noticed on the operated limb in the majority of the dogs. The main complications were the dislocation and loosening of the acetabular cup.

Key words: $\operatorname{dog}$, hip, total prosthesis.

\section{INTRODUÇÃO}

Quando a terapia conservativa para displasia coxofemoral mostra-se ineficaz, é indicado o tratamento cirúrgico. Uma grande variedade de técnicas cirúrgicas é descrita, entretanto nenhuma é considerada ideal (MANLEY, 1998). A prótese total da articulação coxofemoral, por sua vez, é um procedimento que não somente preserva a função do membro como restabelece mecanismos articulares sem dor (OLMSTEAD, 1987).

A prótese total da articulação coxofemoral representa, atualmente, uma das técnicas mais indicadas para o tratamento cirúrgico da displasia coxofemoral severa em cães nos Estados Unidos e na maioria dos países da Europa (SCHULZ et al., 1998; DEARMIN \& SCHULZ, 2004; HARASEN, 2005).

Desde os meados dos anos 70, a prótese total coxofemoral canina tem sido utilizada com sucesso (OLMSTEAD, 1987; WALLACE \& OLMSTEAD, 1995;

'Departamento de Cirurgia e Anestesiologia, Faculdade de Medicina Veterinária e Zootecnia (FMVZ), Universidade Estadual Paulista (UNESP), Botucatu, SP, Brasil. Endereço para correspondência: Avenida Camilo Mazoni, 1064, 18610-285, Jardim Paraíso, Botucatu, SP. E-mail: brunobtu@yahoo.com.br.

IIDepartamento de Ortopedia e Traumatologia da Faculdade de Medicina, UNESP, Botucatu, SP, Brasil.

IIIDepartamento de Reprodução Animal e Radiologia Veterinária, FMVZ, UNESP, Botucatu, SP, Brasil. 
BERGH et al., 2004) e consiste no implante de cúpula acetabular de polietileno de alta densidade e de componente femoral composto de cabeça e haste femoral de aço inoxidável ou titânio. Outros materiais também são utilizados, como a liga metálica de cromo-cobalto (OLMSTEAD et al., 1983; DE YOUNG et al., 1992; WALLACE \& OLMSTEAD, 1995). Existem modelos protéticos não-cimentados, cujos componentes apresentam uma superfície porosa que permite a integração dos mesmos ao tecido ósseo (IDO et al., 1993; OTSUKA et al., 1994); entretanto, as próteses cimentadas são as mais utilizadas na Medicina Veterinária (OLMSTEAD et al., 1981; OLMSTEAD \& SIDEBOTHAM, 1991; MONTGOMERY et al., 1992; REMEDIOS \& FRIES, 1995).

Segundo PIERMATTEI \& FLO (1997), a função total do membro operado é restabelecida ao redor de oito semanas do pós-operatório, sendo considerados fatores como total sustentação de peso, e movimentação sem sinais de dor na articulação coxofemoral. WALLACE \& OLMSTEAD (1995) relatam ainda que, freqüentemente, não se observa dor na reavaliação das articulações coxofemorais dos animais.

O índice de complicações é relativamente baixo, entretanto estas são de difícil resolução (JEHN et al., 2003). As principais complicações são a luxação (OLMSTEAD et al., 1983; WALLACE \& OLMSTEAD, 1995; PRESTON et al., 1999; JEHN et al., 2003; POOYA et al., 2003), o afrouxamento asséptico do componente protético acetabular (LEWIS \& JONES, 1980; DENNY, 1993; PIERMATTEI \& FLO, 1997; SHIELDS et al., 2002), e a infecção (RICHARDSON et al., 1992; DENNY, 1993; DYCE \& OLMSTEAD, 2002).

No Brasil, a técnica ainda é pouco difundida, principalmente por tratar-se de um procedimento que exige importação de componentes protéticos e treinamento especializado. O desenvolvimento de um produto protético nacional permitiria maior disponibilidade de material cirúrgico e eliminaria, em parte, a limitação financeira. Esses fatos, associados ao freqüente diagnóstico de alterações clínicas afetando a articulação coxofemoral observada na clínica de pequenos animais, estimularam o desenvolvimento da presente pesquisa.

Os objetivos deste experimento foram avaliar: a aplicabilidade de prótese total coxofemoral com sistema de cabeça femoral fixa, confeccionada no Brasil, em cães sadios; a evolução pós-operatória dos cães, por meio de exame clínico e radiográfico; e as complicações relacionadas ao procedimento.

\section{MATERIAL E MÉTODOS}

Foram utilizados dez cães (Canis familiaris - Linnaeus, 1758), sem raça definida, machos e fêmeas, adultos, com peso variando entre 18 e $26 \mathrm{~kg}$, sadios e sem alterações no aparelho locomotor.

Todos os animais foram submetidos ao procedimento de prótese total cimentada da articulação coxofemoral esquerda. Constituiu-se um único grupo experimental. Os cães foram mantidos em canis individuais e alimentados com ração comercial e água ad libitum.

O acesso cirúrgico foi realizado por uma abordagem craniolateral da articulação coxofemoral segundo a técnica descrita por PIERMATTEI (1993). A seguir, foi realizada a osteotomia do colo femoral mantendo-se o grau de anteversão. O membro esquerdo dos animais recebeu uma prótese total nacionala, desenvolvida na UNESP- Campus de Botucatu, em conjunto com a Faculdade de Medicina, cimentada, composta por um componente acetabular, confeccionado em polietileno de alta densidade, apresentando sistema retensivo e um componente femoral de cromo-cobalto com cabeça fixa. Três diferentes tamanhos de prótese (pequeno, médio e grande) estavam disponíveis, sendo selecionados de acordo com o porte do animal e a melhor adequação. O acetábulo foi aprofundado, cerca de 5 a $10 \mathrm{~mm}$, com raspa hemisférica específica, removendo-se toda a cartilagem articular. Três ou mais orifícios, nas bordas ilíaca, isquiática e dorsal do acetábulo, foram realizados com auxílio de perfuratriz e broca cirúrgica, a fim de ampliar a superfície de contato entre o cimento ósseo à base de polimetilmetacrilato e o osso. A prótese acetabular foi cimentada, utilizando-se posicionador específico, acompanhando as angulações anatômicas do acetábulo. Uma fresa afilada específica foi utilizada para a preparação do canal femoral; a seguir, a prótese femoral foi cimentada em ângulo neutro de anteversão, acompanhando o eixo transversal dos côndilos femorais. A articulação protética foi reduzida e testada sua movimentação. Os planos teciduais foram suturados com fio de náilon monofilamentar 2-0.

Os animais foram submetidos ao exame clínico, sempre pelo mesmo examinador, nos seguintes momentos após o procedimento cirúrgico: 24 horas (Momento 1- M1); 30 dias (M30); 60 dias (M60); 90 dias (M90); 120 dias (M120); e 150 dias (M150). A função locomotora do membro foi quali-quantificada em escores (AIKEN et al., 1992), e a intensidade de dor classificada segundo tabela modificada por CRUZ et al. (1999).

Todos os animais foram submetidos ao exame radiográfico das articulações coxofemorais, antes e imediatamente após o procedimento cirúrgico (M0), e nos diferentes momentos de avaliação.

Ciência Rural, v.38, n.1, jan-fev, 2008. 


\section{RESULTADO E DISCUSSÃO}

O desenvolvimento de materiais protéticos nacionais é de fundamental importância na difusão da artroplastia canina, pois permitiria maior disponibilidade de material cirúrgico e eliminaria, em parte, a limitação financeira ocasionada pela aplicação desta importante opção terapêutica. Além disso, o estudo continuado e sistemático da sua aplicação possibilitaria a capacitação e o aprimoramento dessa técnica na Medicina Veterinária brasileira.

A displasia coxofemoral canina grave, principal indicação para a realização do procedimento protético (OLMSTEAD et al., 1983; ARIAS et al., 2004), é doença potencialmente causadora de disfunção do membro pélvico em cães (REMEDIOS \& FRIES, 1995; PIERMATTEI \& FLO, 1997; JOHNSON \& HULSE, 2002). As terapias conservativas ou mesmo cirúrgicas não restituem completamente a fisiologia funcional da articulação. A substituição total da articulação por prótese, por sua vez, é um procedimento que, apesar de não restaurar a estrutura anatômica articular normal, preserva a função do membro e restabelece mecanismos articulares sem dor (OLMSTEAD, 1987).

O procedimento de prótese total da articulação coxofemoral, apesar da sua complexidade cirúrgica, foi executado de forma uniforme nos dez cães, seguindo a metodologia proposta, sem complicações significativas. O acompanhamento pós-operatório de seis meses foi suficiente, pois abrange as potenciais complicações observadas no período protético inicial (WALLACE \& OLMSTEAD, 1995; PRESTON et al., 1999; JEHN et al., 2003).

Na presente pesquisa, verificou-se $80 \%$ de índice de sucesso no procedimento de implante protético da articulação coxofemoral. Os resultados encontrados condizem com os relatados na literatura consultada (LEWIS \& JONES, 1980; MONTGOMERY et al., 1992; DENNY, 1993; WARNOCK et al, 2003). Entretanto, cabe mencionar que ainda não se possui ampla experiência cirúrgica e o estabelecimento logístico de aplicação que se observa nos países desenvolvidos e/ou nas instituições referidas.

No primeiro dia do pós-operatório, observou-se início da função locomotora do membro operado, em $70 \%$ dos animais. A partir do $60^{\circ}$ dia de pós-operatório, M60, todos esses cães apresentaram resultado excelente na avaliação clínica. Estes achados estão de acordo com os relatados na literatura (DE YOUNG et al., 1992; JONES, 1994; WALLACE \& OLMSTEAD, 1995; PIERMATTEI \& FLO, 1997), sendo observada a permanência dessa condição nos momentos subseqüentes de avaliação para esses animais. Com relação à intensidade de dor frente à palpação, $60 \%$ dos cães obtiveram ausência de dor aos 60 dias, dado semelhante ao das alterações verificadas no exame da locomoção. Dois animais sofreram luxação e foram os que apresentaram os piores escores. Não foram encontrados na literatura consultada artigos que estudassem diretamente a sensibilidade dolorosa na área adjacente à prótese total da articulação coxofemoral em cães.

Radiograficamente, todos os animais apresentaram posicionamento adequado dos componentes protéticos na avaliação pós-operatória imediata, observando-se encaixe desses componentes e redução protética (Figura 1a). A cimentação pôde ser avaliada pela presença da fina camada de cimento ósseo ao redor dos componentes protéticos (MASSAT \& VASSEUR, 1994). A partir dos 30 dias, notou-se, na maioria dos animais, linha radiolucente de espessura inferior a $1 \mathrm{~mm}$ na interface entre a superfície óssea e o cimento ósseo na região acetabular; este achado é considerado normal (KONDE et al., 1982). Segundo KONDE et al. (1982), a formação dessa linha pode ser justificada pela necrose de osteócitos e substituição por tecido fibroso ou fibrocartilaginoso cicatricial, sendo esta associada à excessiva liberação térmica induzida pela polimerização do cimento ósseo à base de polimetilmetacrilato. A espessura desta linha foi maior que o valor considerado normal somente em um animal; este apresentou a soltura asséptica do componente acetabular, compatível com a alteração notada.

O índice de complicações da prótese total da articulação coxofemoral é relativamente baixo (JEHN \& MANLEY, 2002), ao redor de $18 \%$ dos casos (JONES, 1994); entretanto, pode chegar a até $50 \%$ dos animais submetidos à cirurgia (PALMISIANO et al., 2003). Na presente pesquisa, foram registradas quatro complicações (40\%), índice justificado pela fase inicial de desenvolvimento da cirurgia protética em cães, que pode ter contribuído para que imperfeições, mesmo mínimas, ocorressem, em especial devido à complexidade da técnica, fato semelhante ao relatado na literatura na fase inicial de implantação protética (OLMSTEAD, 1987). Entretanto, as complicações observadas foram tratadas com sucesso nos quatro animais, sendo que dois deles foram submetidos à remoção dos componentes e conversão da técnica em excisão artroplástica.

Os dois casos de luxação da prótese ocorreram nos primeiros dias do pós-operatório. Essa alteração tende a ocorrer dentro de seis semanas após a realização da prótese total da articulação coxofemoral (DYCE et al., 2000). No primeiro momento de avaliação

Ciência Rural, v.38, n.1, jan-fev, 2008. 


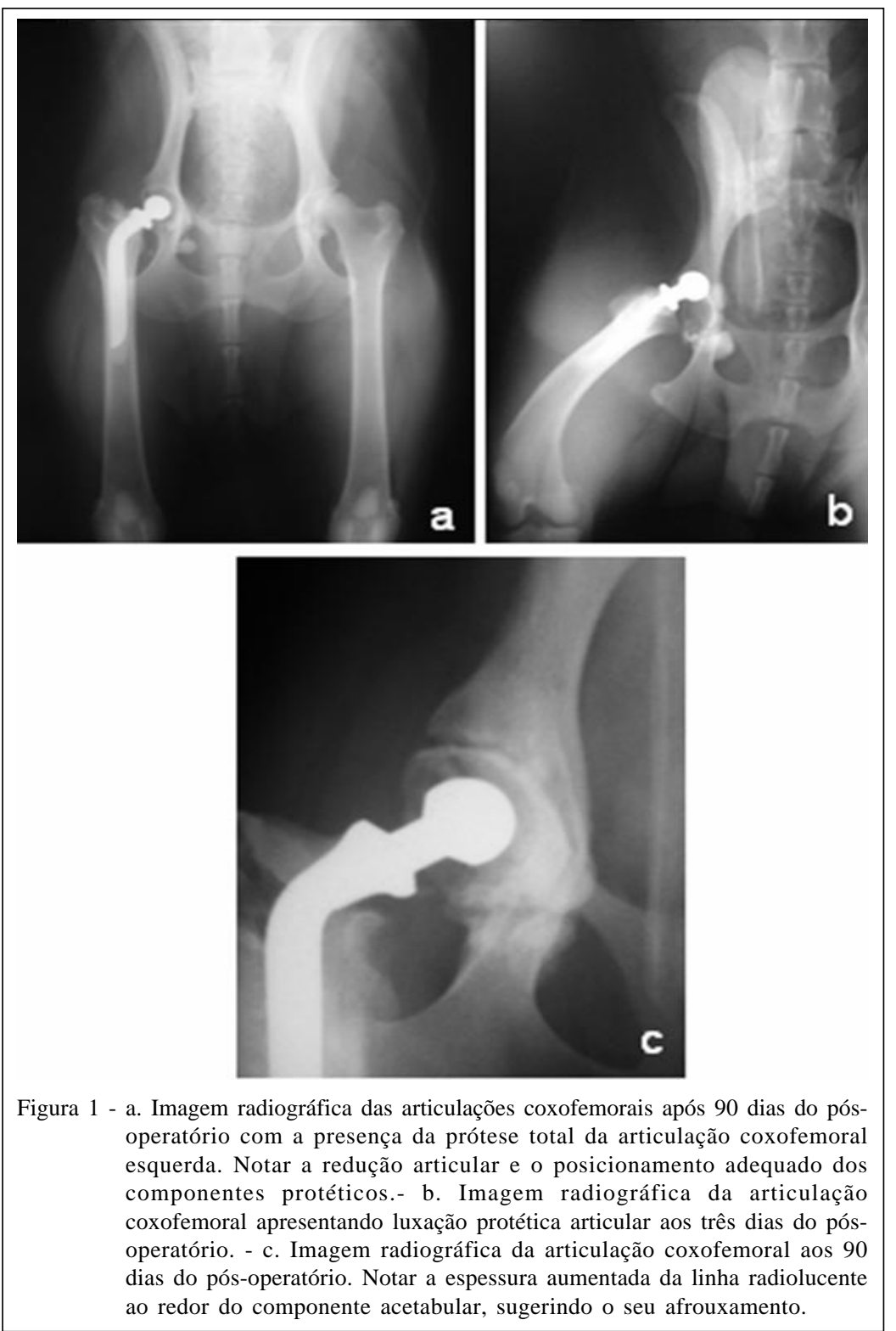

(M1), verificou-se ausência de apoio do membro e presença de dor intensa e mobilidade aumentada. Ao exame radiográfico, confirmou-se a luxação (Figura 1b), sendo sua localização craniodorsal em ambos os animais (C7 e C8). A complicação mais comumente observada após a realização da prótese total da articulação coxofemoral é a luxação ou deslocamento (OLMSTEAD et al., 1983; WALLACE \& OLMSTEAD, 1995; PRESTON et al., 1999; JEHN et al., 2003; POOYA et al., 2003; ARIAS et al., 2004), ocorrendo em 1,1 a $8,5 \%$ dos casos. O posicionamento inadequado dos componentes acetabular e femoral está intimamente relacionado com os mecanismos dessa complicação (JEHN et al., 2003), apesar de haver uma grande associação entre a luxação da prótese coxofemoral canina e algum evento traumático (OLMSTEAD et al., 1983; CROSS et al., 2000). A escolha inadequada do comprimento do colo femoral também pode determinar um risco mais elevado (SCHULZ, 2000). Ambos os animais que sofreram luxação da articulação protética apresentavam deficiência discreta na cobertura acetabular dorsal, o que pode ter predisposto o deslocamento dorsal, como citado por MASSAT \& VASSEUR (1994). Entretanto, não se pode descartar a ocorrência de um evento traumático, visto que os animais não foram acompanhados durante o período noturno, apesar de estarem confinados em jaulas. A ausência de restrição adequada de atividades no 
período pós-operatório é outro fator associado para a ocorrência da luxação (OLMSTEAD, 1995).

O melhor método de tratamento para os casos de luxação é a redução aberta (OLMSTEAD, 1987); entretanto, nas luxações cuja causa é o posicionamento inadequado dos componentes protéticos, estes devem ser removidos e reposicionados (DYCE et al., 2000). Por considerar a alteração no posicionamento dos componentes discreta, optou-se pela redução aberta da luxação sem reposicionamento. A recuperação satisfatória dos animais, após o procedimento de revisão e a conseqüente redução da luxação, reafirma a ausência de alterações posicionais importantes nos componentes protéticos.

Outros dois cães (C2 e C10) apresentaram afrouxamento do componente acetabular e foram submetidos ao procedimento de conversão da técnica de prótese total em excisão artroplástica da cabeça e do colo femoral, como descrito por DYCE \& OLMSTEAD (2002). O primeiro (C2) apresentou característica asséptica de soltura, com dificuldade de locomoção do membro posterior esquerdo, logo nos primeiros dias do pós-operatório. Após 90 dias, verificou-se claudicação discreta, presença de dor e crepitação na palpação da articulação coxofemoral. Após diagnóstico com auxílio do exame radiográfico (Figura 1c), observou-se, na artrotomia exploratória, aos 100 dias do pós-operatório, a soltura do componente acetabular na sua interface óssea, sem sinais macroscópicos de infecção; a ausência desta foi confirmada pela cultura microbiológica negativa. No segundo animal (C10), por sua vez, foi evidenciada a soltura séptica do componente acetabular, sendo verificado o crescimento de Staphylococcus aureus $\hat{a}$ hemolitico. Após a excisão artroplástica da cabeça e do colo femorais, os animais apresentaram melhora significativa e, aos 150 dias do pós-operatório, observou-se claudicação muito discreta e ausência de dor na palpação coxofemoral.

O afrouxamento dos componentes protéticos é uma importante complicação observada na prótese total coxofemoral em cães (SHIELDS et al., 2002) e, freqüentemente, resulta em remoção dos componentes e conseqüente insucesso do procedimento (WALLACE \& OLMSTEAD, 1995). Segundo POOYA et al. (2003), o afrouxamento asséptico do componente acetabular está associado com causas mecânicas, especialmente devido ao seu posicionamento inadequado (OLMSTEAD et al., 1983), o qual desencadeia uma distribuição desigual de forças sobre o componente, causando colapso progressivo do cimento (POOYA et al., 2003). Uma alteração mesmo que discreta no posicionamento dos componentes pode desencadear a soltura dos mesmos, e isto pode ter sido um fator a ser considerado no afrouxamento asséptico no presente experimento. Associado a isso, deve-se acrescentar a presença de comportamento agitado do animal (C2).

No cão 10, a suspeita da infecção ocorreu no primeiro mês do pós-operatório. Segundo DYCE \& OLMSTEAD (2002), a origem da infecção na fase inicial do pós-operatório é verificada, normalmente, por contaminação durante o procedimento protético ou extensão da ferida cirúrgica, visto que as hematógenas são observadas no pós-operatório tardio e consideradas raras em cães. Além disso, segundo KONDE et al. (1982), há uma associação entre a ocorrência de afrouxamento dos componentes protéticos posteriormente à infecção dos mesmos. A soltura do componente acetabular é uma importante complicação após a realização da prótese total e, por apresentar difícil resolução, deve ser evitada. Contudo, a conversão do procedimento para a excisão artroplástica é opção terapêutica satisfatória nesses casos (DYCE \& OLMSTEAD, 2002).

A prótese total da articulação coxofemoral apresenta usualmente resultados excelentes com função satisfatória durante toda a vida do animal. Isso faz desta técnica um procedimento clínico confiável para o tratamento de uma variedade de condições anormais da articulação coxofemoral (PIERMATTEI \& FLO, 1997), em especial da displasia coxofemoral canina, que, nos dias atuais, é uma afecção freqüentemente observada na clínica de pequenos animais em todo o mundo. Infelizmente, o alto custo da prótese importada e a pouca difusão da técnica limitaram e ainda limitam sua aplicação no Brasil.

\section{CONCLUSÃO}

A prótese total coxofemoral com sistema de cabeça femoral fixa, confeccionada no Brasil, proporciona locomoção satisfatória sem claudicação na maioria dos cães (80\%), observando-se melhora progressiva da locomoção e da sensibilidade dolorosa, com a evolução do pós-operatório, tornando-a clinicamente aplicável em cães. As principais complicações relacionadas ao procedimento são a luxação da articulação coxofemoral protética e a soltura do componente acetabular.

\section{AGRADECIMENTOS}

À Fundação de Amparo à Pesquisa do Estado de São Paulo (Fapesp), pela concessão da bolsa de estudos, e à empresa Cruz Alta Pro-Hospitalar Ltda., pelo apoio essencial para a realização do projeto de pesquisa. 


\section{ASPECTOS ÉTICOS}

Os procedimentos utilizados foram aprovados pela Câmara de Ética em Experimentação Animal da Faculdade de Medicina Veterinária e Zootecnia - UNESP - Campus de Botucatu. Os cães foram doados após o término da avaliação pós-operatória.

\section{FONTE DE AQUISIÇÃO}

a - Cruz Alta Pro-Hospitalar Ltda - Fernadópolis - SP.

\section{REFERÊNCIAS}

AIKEN, S.W. et al. Extra-articular fascial strip repair of the cranial cruciate deficient stifle: technique and results in seven dogs. Vet Comp Orthop Traumatol, v.5, p.145-150, 1992.

ARIAS, S.A. et al. Prótese total da articulação coxofemoral em cães: Relato de dois casos. Arq Bras Med Vet Zootec, v.56, n.5, p.618-622, 2004.

BERGH, M.S. et al. Femoral bone adaptation to unstable longterm cemented total hip arthroplasty in dogs. Vet Surg, v.33, n.3, p.238-245, 2004.

CROSS, A.R. et al. Acetabular component orientation as an indicator of implant luxation in cemented total hip arthroplasty. Vet Surg, v.29, p.517-523, 2000.

CRUZ, M.L. et al. Efeitos do flunixin, ketoprofeno, carprofeno, buprenorfina e placebo para a analgesia pós-operatória em cães submetidos à osteossíntese de fêmur. A Hora Vet, p.1-17, 1999.

De YOUNG, D.J. et al. Implantation of uncemented total hip prosthesis. Technique and initial results of 100 arthroplasties. Vet Surg, v.21, n.3, p.168-177, 1992.

DEARMIN, M.G.; SCHULZ, K.S. The effect of stem length on femoral component positioning in canine total hip arthroplasty. Vet Surg, v.33, p.272-278, 2004.

DENNY, H.R. The hindlimb. In: A guide to canine and feline orthopaedic surgery. 3.ed. Oxford: Blackwell, 1993. Cap.5, p.284-388.

DYCE, J. et al. Evaluation of risk factors for luxation after total hip replacement in dogs. Vet Surg, v.29, n.6, p.524532, 2000.

DYCE, J.; OLMSTEAD, M.L. Removal of infected canine cemented total hip prostheses using a femoral window technique. Vet Surg, v.31, p.552-560, 2002.

HARASEN, G. Orthopedics odds and ends. Can Vet J, v.46, n.2, p.170-171, 2005.

IDO, K. et al. Cementless total hip replacement. Acta Orthop Scand, v.64, n.6, p.607-612, 1993.

JEHN, C.T.; MANLEY, P.A. The effects of femur and implant position on the radiographic assessment of total hip femoral implants in dogs. Vet Surg, v.31, p.349-357, 2002.
JEHN, C.T. et al. Orthogonal view analysis for evaluating the femoral component position of total hip implants in dogs using postoperative radiographs. Vet Surg, v.32, p.134-141, 2003.

JOHNSON, A.L.; HULSE, D.A. Diseases of the joints. In: FOSSUM, T.W. Small animal surgery. 2.ed. St. Louis: Mosby, 2002. Cap.35, p.1023-1157.

JONES, D.G.C. The hip joint. In: HOULTON, J; COLLINSON, R. Manual of small animal arthrology. Bournemouth: Grafos, 1994. Cap.15, p.243-266.

KONDE, L.J. et al. Radiographic evaluation of total hip replacement in the dog. Vet Radiology, v.23, n.3, p.98-106, 1982.

LEWIS, R.H.; JONES JR., J.P. A clinical study of canine total hip artroplasty. Vet Surg, v.9, p.20-23, 1980.

MANLEY, P.A. Articulação coxofemoral. In: SLATTER, D. Manual de cirurgia de pequenos animais. 2.ed. São Paulo: Manole, 1998. Cap.135, p.2113-2134.

MASSAT, B.J.; VASSEUR, P.B. Clinical and radiographic results of total hip arthroplasty in dogs: 96 cases (1986-1992). J Am Vet Med Assoc, v.205, n.3, p.448-454, 1994.

MONTGOMERY, R.D. et al. Total hip arthroplasty for treatment of canine hip dysplasia. Vet Clin North Am: Small Anim Pract, v.22, n.3, p.703-719, 1992.

OLMSTEAD, M.L. The canine cemented modular total hip prosthesis. J Am Anim Hosp Assoc, v.31, p.109-123, 1995

OLMSTEAD, M.L. Total hip replacement. Vet Clin North Am: Small Anim Pract, v.17, n.4, p.943-954, 1987.

OLMSTEAD, M.L. et al. A five-year study of 221 total hip replacement in the dog. Am J Vet Med Assoc, v.183, n.2, p.191-194, 1983.

OLMSTEAD, M.L. et al. Technique for canine total hip replacement. Vet Surg, v.10, p.44-50, 1981.

OLMSTEAD, M.L.; SIDEBOTHAM, C. Premiliary report on a new canine modular total hip. Vet Surg, v.20, p.343, 1991.

OTSUKA, N.Y. et al. Fixation with biodegradable devices of acetabular components in a canine model. Clin Orthop, v.306, p.250-255, 1994.

PALMISIANO, M.P. et al. Extraosseous cement granuloma associated with total hip replacement in 6 dogs. Vet Surg, v.32, p.80-90, 2003.

PIERMATTEI, D.L. The pelvis and hip joint. In: An atlas of surgical approaches to the bones and joints of the dog and cats. 3.ed. Philadelphia: Saunders, 1993. Cap.6, p.221-265.

PIERMATTEI, D.L.; FLO, G.L. A articulação coxofemoral. In: BRINKER, W.O. et al. Ortopedia e tratamento das fraturas dos pequenos animais. 3.ed. São Paulo: Manole, 1997. Cap.15, p.394-436. 
POOYA, H.A. et al. Short-term evaluation of dorsal acetabular augmentation im 10 canine total hip replacement. Vet Surg, v.32, p.142-152, 2003

PRESTON, C.A. et al. Total hip artroplasty in nine canine hind limb amputees: a retrospective study. Vet Surg, v.28, p.341-347, 1999.

REMEDIOS, A.M.; FRIES, C.L. Treatment of canine hip dysplasia: A review. Can Vet J, v.36, p.503-509, 1995.

RICHARDSON, D.C. et al. Pharmacokinetic disposition of cefazolin in serum and tissue during canine total hip replacement. Vet Surg, v.21, n.1, p.1-4, 1992.

SCHULZ, K.S. Application of arthrosplasty principles to canine cemented total hip replacement. Vet Surg, v.29, p.578-593, 2000 .
SCHULZ, K.S. et al. Transverse plane evaluation of the effects of surgical technique on stem positioning and geometry of reconstruction in canine total hip replacement. Am J Vet Res, v.59, n.8, p.1071-1079, 1998.

SHIELDS, S.L. et al. The effects of acetabular cup temperature and duration of cement pressurization on cement porosity in a canine total hip replacement model. Vet Surg, v.31, p.167173, 2002.

WALLACE, L.J.; OLMSTEAD, M.L. Disabling conditions of canine coxofemoral joint. In: OLMSTEAD, M.L. Small animal orthopedics. St. Louis: Mosby, 1995. Cap.17, p.361394.

WARNOCK, J.J. et al. Retrospective analysis of canine miniature total hip prostheses. Vet Surg, v.32, p.285-291, 2003. 